\title{
Augmented Invariance Control for Systems with Smoothness Constraints
}

\author{
Melanie Kimmel, Christoph Jähne and Sandra Hirche
}

\begin{abstract}
In many applications, the controlled dynamical system is subject to constraints, e.g. to enforce a desired performance. There are various methods, which guarantee the enforcement of such constraints. They do, however, not provide satisfactory solutions if the system state has to exhibit certain smoothness properties. This work presents a novel method of designing a control input, which guarantees the adherence to constraints as well as a desired smoothness of the system state. The control scheme is implemented as an add-on to an existing task dependent control law. Augmentation of the dynamical system achieves a desired smoothness of the state whereas methods from invariance control are utilized to guarantee constraint enforcement.
\end{abstract}

\section{INTRODUCTION}

In control applications the system behavior is often subject to constraints. These may either be internal, e.g. inherited from the system structure, or external, e.g. caused by the control task or the environment. Safety specifications are another such example. They are especially important whenever humans are involved, e.g. in human-in-the-loop control designs [1], in order to avoid unnecessary danger. Applications include modern cars, where humans share the control of the vehicle with the internal controllers and industrial and domestic environments, where robots collaborate with humans and are even used for rehabilitation purposes. Due to this interconnection, the control input should be designed on the one hand to enforce the necessary safety constraints but on the other hand with the appropriate smoothness required for the overall control task.

Naturally, there exist various methods for constraint enforcement. Approaches based on potential functions [2] enforce state and output constraints. Model predictive control [3] and the command governor approach [4] guarantee the enforcement of input, output and state constraints by solving a constrained optimization problem. Barrier certificates are also used to verify safety guarantees [5] and are included as control barrier functions in control structures, e.g. by using them as constraints in a convex optimization [6]. Invariance control [7] solves a similar convex optimization problem, but encodes the constraints in an invariance function derived from the system dynamics. None of these approaches, however, provide a solution on how to design the control input with respect to smoothness and dynamic properties. Without additional constraints on the input or penalty terms in the cost function, the approaches are prone

All authors are with the Chair of Information-Oriented Control, Department of Electrical Engineering and Information Technology, Technical University of Munich, D-80290 München, Germany. \{melanie.kimmel, c.jaehne, hirche\}etum.de to discontinuities in the control signal whenever a constraint is added or removed. In general, it is not possible to resolve this issue by adding hard input constraints to account for the desired properties as this may render the problem infeasible and by adding soft constraints, the desired nominal control dynamics may not be achieved.

In this work, a novel method is presented, which controls a dynamical system to enforce constraints as well as a desired smoothness of the state. The desired dynamic properties are encoded in an additional dynamical system augmenting the original dynamics. A solution is provided to adapt the task enforcing control structure accordingly. A constraint enforcing control scheme is then designed based on the methods from invariance control as an add-on to the augmented system. The approach is shown to guarantee constraint enforcement by rendering the augmented system invariant w.r.t a constraint admissible set of states, while preserving the stability of the nominally controlled original system. A numerical example demonstrates the characteristics of the approach.

The remainder of this paper is organized as follows: In Section II the requirements are summarized and invariance control is introduced. In Section III the novel method with augmented input dynamics is discussed. Section IV illustrates the novel approach in a numerical example. Concluding remarks are given in Section V.

Notation: Vectors are represented by lower case bold letters and matrices by capital bold letters. The Euclidean norm of a vector $\boldsymbol{x}$ is expressed by $\|\boldsymbol{x}\|_{2}=\sqrt{\boldsymbol{x}^{\top} \boldsymbol{x}}$. Derivatives w.r.t. time are indicated by $\dot{x}:=\frac{d x}{d t}$ and $x^{(v)}:=\frac{\mathrm{d}^{v} x}{\mathrm{~d} t^{v}}$. The Lie-derivative of a scalar function $h(\boldsymbol{x}) \in \mathbb{R}$ with $\boldsymbol{x} \in \mathbb{R}^{n}$ along a vector field $\boldsymbol{f}(\boldsymbol{x}): \mathbb{R}^{n} \rightarrow \mathbb{R}^{n}$ or a matrix vector field $\boldsymbol{G}(\boldsymbol{x}): \mathbb{R}^{n} \rightarrow \mathbb{R}^{n \times m}$ is expressed by

$$
\mathcal{L}_{\boldsymbol{f}} h(\boldsymbol{x})=\frac{\partial h(\boldsymbol{x})}{\partial \boldsymbol{x}} \boldsymbol{f}(\boldsymbol{x}) \quad \text { and } \quad \mathcal{L}_{\boldsymbol{G}} h(\boldsymbol{x})=\frac{\partial h(\boldsymbol{x})}{\partial \boldsymbol{x}} \boldsymbol{G}(\boldsymbol{x}) .
$$

Lie-derivatives $\mathcal{L}_{\boldsymbol{f}}^{v} h(\boldsymbol{x})$ with $v \in \mathbb{N}$ are defined recursively. The set $\mathcal{C}^{v}$ denotes the set of continuous functions that are $v$ times continuously derivable w.r.t. to time.

\section{BACKGROUND}

This section summarizes the general approach of enforcing constraints using invariance control as introduced in [7], [8].

\section{A. System and Constraints}

We consider a nonlinear, time-invariant, control affine system with state $\boldsymbol{x} \in \mathbb{R}^{n}$, input $\boldsymbol{u} \in \mathbb{R}^{m}$ and output $\boldsymbol{y} \in \mathbb{R}^{q}$.

$$
\left.\begin{array}{rl}
\dot{\boldsymbol{x}} & =\boldsymbol{f}(\boldsymbol{x})+\boldsymbol{G}(\boldsymbol{x}) \boldsymbol{u} \\
\boldsymbol{y} & =\boldsymbol{h}(\boldsymbol{x})
\end{array}\right\}
$$


The vector field $\boldsymbol{f}: \mathbb{R}^{n} \rightarrow \mathbb{R}^{n}$ and the matrix vector field $\boldsymbol{G}=\left[\boldsymbol{g}_{1} \ldots \boldsymbol{g}_{m}\right]: \mathbb{R}^{n} \rightarrow \mathbb{R}^{n} \times \mathbb{R}^{m}$ define the dynamics. A nominal input $\boldsymbol{u}_{\text {no }}$ enforces performance, stability and the control goal without consideration of constraints. The design of such a nominal control scheme is not the focus of this work and it is assumed to exist. In addition, the system (1) is subject to static state and/or output constraints

$$
h_{\mathrm{c}, i}(\boldsymbol{x}) \leq 0, \quad 1<i<l .
$$

By definition, a state of (1) is constraint admissible, if it fulfills (2), which yields the notion of the admissible set

$$
\mathcal{H}(\boldsymbol{x})=\left\{\boldsymbol{x} \in \mathbb{R}^{n} \mid h_{\mathrm{c}, i}(\boldsymbol{x}) \leq 0, \forall 1<i<l\right\} .
$$

Naturally, time-dependent constraints may also be defined. For ease of notation, we consider static bounds, but the approach may easily be extended using the methods from [9].

\section{B. Invariance Control}

Invariance control generates a switching control law, which is implemented as an add-on to nominal control to guarantee constraint enforcement. Figure 1 shows a schematic of the resulting control loop. Essentially, invari-

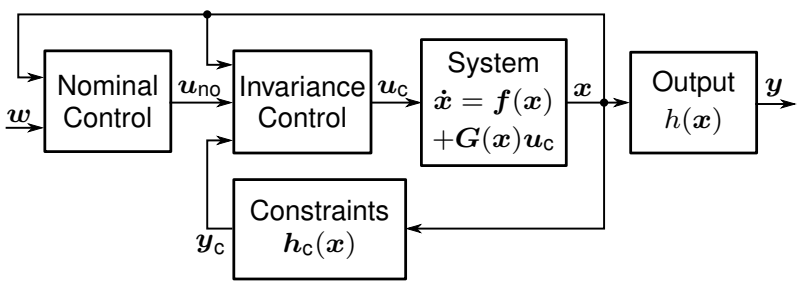

Fig. 1: Nonlinear dynamical system controlled by a combination of task enforcing nominal control with a desired state trajectory $\boldsymbol{w}$ and constraint enforcing invariance control.

ance control [7], [8] supervises the constraints and changes the control input only when it is inevitable to avoid constraint violation. It is based on deriving conditions on the input $\boldsymbol{u}$. First, a constraint output is defined using (2).

$$
\boldsymbol{y}_{\mathrm{c}}=\boldsymbol{h}_{\mathrm{c}}(\boldsymbol{x})
$$

Then a correlation between $\boldsymbol{u}$ and $\boldsymbol{y}_{\mathrm{c}}$ is determined using input/output (I/O)-linearization. A necessary and sufficient condition for the existence of a I/O-linearizing transformation is a well-defined vector relative degree [10].

Definition 1: The system (1) has a well-defined vector relative degree $\left(r_{1}, \ldots, r_{l}\right)$ on a subset $\mathcal{X}$ of the state space if the following conditions are fulfilled for all $\boldsymbol{x} \in \mathcal{X} \subseteq \mathbb{R}^{n}$ :

1) $\mathcal{L}_{\boldsymbol{G}} \mathcal{L}_{\boldsymbol{f}}^{k} h_{\mathrm{c}, i}(\boldsymbol{x})=\mathbf{0} \forall 0 \leq k<r_{i}-1,1 \leq i \leq l$,

2) the decoupling matrix has full row rank.

$$
\operatorname{rank}(\boldsymbol{A}(\boldsymbol{x}))=\operatorname{rank}\left(\left[\begin{array}{c}
\mathcal{L}_{\boldsymbol{G}} \mathcal{L}_{\boldsymbol{f}}^{r_{1}-1} h_{\mathrm{c}, 1}(\boldsymbol{x}) \\
\vdots \\
\mathcal{L}_{\boldsymbol{G}} \mathcal{L}_{\boldsymbol{f}}^{r_{l}-1} h_{\mathrm{c}, l}(\boldsymbol{x})
\end{array}\right]\right)=l
$$

Obviously, if the number of constraints is larger than the input dimension $l>m$, the second condition is not fulfilled. This issue will be addressed briefly after the determination of corrective control is introduced.
The input transformation, resulting from the I/Olinearization with respect to one constraint is given by

$$
h_{\mathrm{c}, i}^{\left(r_{i}\right)}(\boldsymbol{x})=\boldsymbol{a}_{i}^{\boldsymbol{\top}}(\boldsymbol{x}) \boldsymbol{u}+b_{i}(\boldsymbol{x})
$$

$$
\text { with } \boldsymbol{a}_{i}^{\boldsymbol{\top}}(\boldsymbol{x})=\mathcal{L}_{\boldsymbol{G}} \mathcal{L}_{\boldsymbol{f}}^{r_{i}-1} h_{\mathrm{c}, i}(\boldsymbol{x}), b_{i}(\boldsymbol{x})=\mathcal{L}_{\boldsymbol{f}}^{r_{i}} h_{\mathrm{c}, i}(\boldsymbol{x})
$$

and the relative degree $r_{i}$ of $h_{i}(\boldsymbol{x})$ w.r.t. $\boldsymbol{u}$. The transformation exists for each output independently if $y_{\mathrm{c}, i}=h_{\mathrm{c}, i}(\boldsymbol{x})$ has a well-defined vector relative degree according to Def. 1. Setting the new input

$$
z_{i}=h_{\mathrm{c}, i}^{\left(r_{i}\right)}(\boldsymbol{x})
$$

to a constant negative value eventually leads to a decrease in the output $y_{\mathrm{c}, i}=h_{\mathrm{c}, i}(\boldsymbol{x})$, since $z_{i}$ and $y_{\mathrm{c}, i}$ are connected through an integrator chain. Choosing $z_{i} \leq \gamma_{i}<0$, where $\gamma_{i}<0$ is a constant design parameter, introduces an upper bound on the future output values $\boldsymbol{h}_{\mathrm{c}}(t+\Delta t)=$ $\boldsymbol{h}_{\mathrm{c}}(\boldsymbol{x}(t+\Delta t))$ with $\Delta t \geq 0$.

$$
h_{\mathrm{c}, i}(t+\Delta t) \leq \frac{\Delta t^{r_{i}}}{r_{i} !} \gamma_{i}+\sum_{j=0}^{r_{i}-1} \frac{\Delta t^{j}}{j !} h_{\mathrm{c}, i}^{(j)}(t)=p_{i}(t, \Delta t)
$$

This motivates the so-called invariance function [7]

$$
\Phi_{i}\left(\boldsymbol{x}, \gamma_{i}\right)=\underset{\Delta t \geq 0}{\operatorname{argmax}} p_{i}(t, \Delta t),
$$

which represents the maximum future value of $y_{\mathrm{c}, i}$ if $z_{i} \leq$ $\gamma_{i}<0$ is applied from $t$ onwards. As the goal of invariance control is to keep the state of (1) within the admissible set of states (3), a value of $\Phi_{i}\left(\boldsymbol{x}, \gamma_{i}\right)=0$ indicates a possible necessity of corrective control as for $z_{i}>\gamma_{i}$ the maximum future value of $y_{\mathrm{c}, i}$ violates the constraint. Thus by designing a control law, which keeps the state within the invariant set

$$
\mathcal{G}(\boldsymbol{x})=\left\{\boldsymbol{x} \in \mathbb{R}^{n} \mid \Phi_{i}\left(\boldsymbol{x}, \gamma_{i}\right) \leq 0 \quad \forall 1 \leq i \leq l\right\}
$$

the state stays within the admissible set (3). Based on the invariance function, the set of active constraints

$$
\mathcal{K}(\boldsymbol{x})=\left\{i \in\{1,2, \ldots, l\} \mid \Phi_{i}\left(\boldsymbol{x}, \gamma_{i}\right) \geq 0\right\},
$$

i.e. constraints that are in danger of being violated, is defined and the switching control law is introduced

$$
z_{\mathrm{c}, i}= \begin{cases}\min \left(\gamma_{i}, z_{\mathrm{no}, i}\right) & \text { if } \Phi_{i}\left(\boldsymbol{x}, \gamma_{i}\right) \geq 0 \\ z_{\mathrm{no}, i} & \text { else }\end{cases}
$$

with $z_{\mathrm{no}, i}=\boldsymbol{a}_{i}^{\boldsymbol{\top}}(\boldsymbol{x}) \boldsymbol{u}_{\mathrm{no}}+b_{i}(\boldsymbol{x})$ being the nominal input of the I/O-linearized system. The switching ensures that a corrective control value $z_{\mathrm{c}, i} \leq \gamma_{i}$ is applied whenever a constraint is active. Combining (5), (6) and (11) yields

$$
\boldsymbol{a}_{i}^{\boldsymbol{\top}}(\boldsymbol{x}) \boldsymbol{u}+b_{i}(\boldsymbol{x}) \leq z_{\mathrm{c}, i} \quad \forall i \in \mathcal{K}(\boldsymbol{x}),
$$

which ensures adherence to the active constraints. As nominal control suffices for inactive constraints, they do not impose constraints on $\boldsymbol{u}$. Corrective control $\boldsymbol{u}_{\mathrm{c}} \in \mathbb{R}^{m}$ is then determined by

$$
\begin{gathered}
\boldsymbol{u}_{\mathrm{c}}=\underset{\boldsymbol{u}}{\operatorname{argmin}}\left\|\boldsymbol{u}-\boldsymbol{u}_{\mathrm{no}}\right\|_{2}^{2} \\
\text { s.t. (12), }
\end{gathered}
$$


which ensures $\boldsymbol{u}_{\mathrm{c}}=\boldsymbol{u}_{\mathrm{no}}$ whenever no constraints are active. For active constraints the difference between $\boldsymbol{u}_{\mathrm{c}}$ and $\boldsymbol{u}_{\text {no }}$ is minimized in the Euclidean sense to achieve a behavior that is as close as possible to the nominal control task. Note that (13) is a convex optimization problem with convex constraints, as they are linear in $\boldsymbol{u}$. Therefore, some of the constraints hold with equality and for the others hold with strict inequality $\boldsymbol{a}_{i}^{\top}(\boldsymbol{x}) \boldsymbol{u}+b_{i}(\boldsymbol{x})<z_{\mathrm{c}, i}$ in the solution [11]. As $\boldsymbol{u} \in \mathbb{R}^{m}$, the solution is uniquely defined by $k \leq m$ equality constraints with linearly independent $\boldsymbol{a}_{i}^{\top}(\boldsymbol{x})$. In the following, the set $\mathcal{K}_{\mathcal{I}}$ denotes these constraints, which fulfill

$$
\left.\begin{array}{l}
\boldsymbol{a}_{i}^{\top}(\boldsymbol{x}) \boldsymbol{u}+b_{i}(\boldsymbol{x})=z_{c, i} \\
\operatorname{rank}\left(\boldsymbol{A}_{\mathcal{K}_{\mathcal{I}}}\right)=\left|\mathcal{K}_{\mathcal{I}}\right|, \boldsymbol{A}_{\mathcal{K}_{\mathcal{I}}}=\left[\boldsymbol{a}_{i}, \ldots\right]^{\top}
\end{array}\right\} \forall i \in \mathcal{K}_{\mathcal{I}} .
$$

The remaining $m-k$ degrees of freedom are determined by the minimum Euclidean distance to $\boldsymbol{u}_{\mathrm{no}}$. An output consisting only of the constraints in $\mathcal{K}_{\mathcal{I}}$ fulfills the second condition of Def. 1 as the $\boldsymbol{a}_{i}^{\top}(\boldsymbol{x})$ are linearly independent, resulting in a well-defined relative degree and thus making the I/Olinearization feasible. Application of $\boldsymbol{u}_{\mathrm{c}}$ renders the state $\boldsymbol{x}$ controlled positive invariant with respect to the invariant set (9), i.e. once the state enters the invariant set it stays within for all future times [8]. Note that, if the total relative degree $r$ with $r=\sum_{i \in \mathcal{K}_{\mathcal{I}}} r_{i}$ is less than the number of states $n$, internal dynamics occur, the stability of which has to be investigated separately.

Although the switching control law guarantees constraint enforcement, it leads to input discontinuities whenever a constraint becomes in-/active. Figure 2 shows an example for a constrained double integrator. Every couple of time

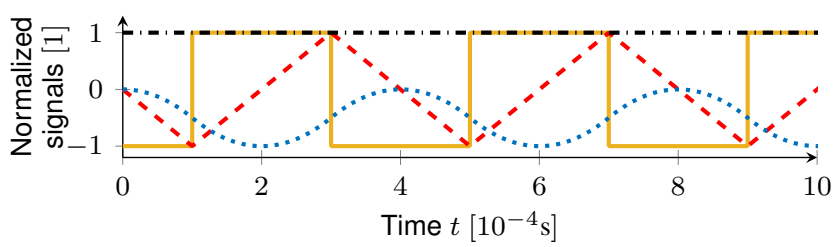

Fig. 2: Invariance controlled integrator chain $\dot{x}_{1}=x_{2}$, $\dot{x}_{2}=u$ with the constraint $x_{1} \leq 0$ and the nominal control $u_{\text {no }}=-\left(x_{1}-1\right)$. Depicted are the normalized signals $\cdots \cdots x_{1} / 10^{-8},--x_{2} / 10^{-4}, \simeq u / 1$ and $\cdots \cdots u_{\mathrm{no}} / 1$. steps, the input switches from nominal to corrective control and vice versa, resulting in a discontinuity. This causes unnecessary stress of the system and a continuous but not continuously differentiable state $\boldsymbol{x}(t) \in \mathcal{C}^{0}$, which is evident from the inflection points in $x_{2}$. This is, however, unwanted in applications requiring a smoother state $\boldsymbol{x}(t) \in \mathcal{C}^{v}$ such as the envisioned human-in-the-loop control design.

\section{Smoothness Design by Augmentation}

Whenever a certain dynamic behavior of the constraint enforcing input is desired, standard invariance control is unsuitable due to the switching characteristics. This section introduces a novel approach, which moves the switching to a higher order time derivative of the control input. The basic idea is to augment (1) by an additional dynamic structure constituting the input dynamics. Invariance control is then designed for the augmented system. The desired structure is shown in Fig. 3. The augmentation poses, however, some

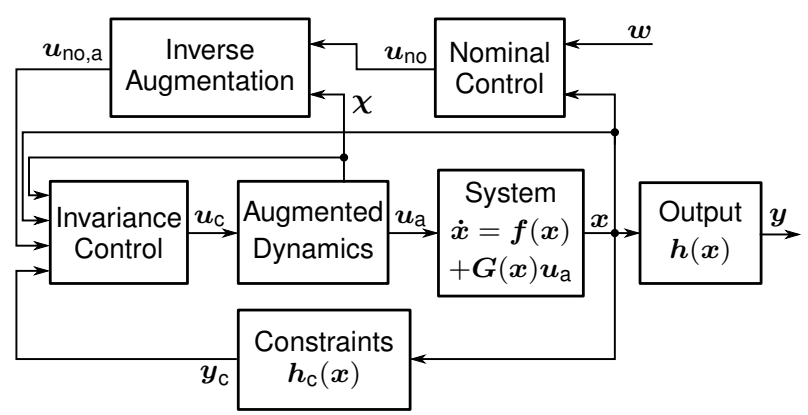

Fig. 3: Nonlinear dynamical system controlled by augmented invariance control combining nominal control, input dynamics and constraint enforcing control.

challenges on the nominal control design, as in the unconstrained case, the augmented system should display the same behavior as the original dynamics under nominal control.

In the following derivation, we assume that switching invariance control according to [7], [8] is able to guarantee the enforcement of the constraints (4) on the original dynamics (1).

Assumption 1: The vector fields $\boldsymbol{g}_{i}(\boldsymbol{x}), 1 \leq i \leq m$ and $\boldsymbol{f}(\boldsymbol{x})$ are Lipschitz continuous in $\boldsymbol{x}$.

Assumption 2: The admissible set $\mathcal{H}(\boldsymbol{x})$ as defined in (3) is not empty.

Assumption 3: Each single output $y_{\mathrm{c}, i}=h_{\mathrm{c}, i}(\boldsymbol{x})$ has a well-defined vector relative degree according to Def. 1 .

Assumption 4: I/O-linearization of (1) with respect to the constraints in $\mathcal{K}_{\mathcal{I}}$ fulfilling (14) yields input-to-state stable (ISS) internal dynamics.

Ass. 1 is usually fulfilled at least locally by dynamics derived from real systems and ensures the existence of a unique solution of the state trajectory $\boldsymbol{x}(t)$ of (1). As a successful enforcement of the constraints is only possible if the constraints are not contradicting, Ass. 2 is a reasonable condition. Ass. 3 is a condition on the controllability of the dynamics and ensures the existence of corrective control while Ass. 4 guarantees the stability of the invariance controlled system.

\section{A. Augmented Dynamics}

The augmented dynamics are defined as a nonlinear, control affine system of the form

$$
\left.\begin{array}{rl}
\dot{\chi} & =f_{\mathrm{a}}(\chi)+\boldsymbol{G}_{\mathrm{a}}(\chi) \boldsymbol{u}_{\mathrm{c}} \\
\boldsymbol{u}_{\mathrm{a}} & =\boldsymbol{h}_{\mathrm{c}, \mathrm{a}}(\chi),
\end{array}\right\}
$$

where $\chi \in \mathbb{R}^{m \cdot v}$ is the state, $\boldsymbol{u}_{\mathrm{a}} \in \mathbb{R}^{m}$ is the control input of (1), $\boldsymbol{u}_{\mathrm{c}} \in \mathbb{R}^{m}$ is the control input of the augmentation and the vector and matrix vector fields $\boldsymbol{f}_{\mathrm{a}}: \mathbb{R}^{m \cdot v} \rightarrow \mathbb{R}^{m}$ and $\boldsymbol{G}_{\mathrm{a}}: \mathbb{R}^{m \cdot v} \rightarrow \mathbb{R}^{m \times m}$ are sufficiently smooth. The goal of the augmentation is a state $\boldsymbol{x} \in \mathcal{C}^{v}$.

Assumption 5: I/O-linearization of the augmentation (15) with respect to the output $\boldsymbol{u}_{\text {a }}$ yields a vector relative de- 
gree $\left(r_{\mathrm{a}, 1}, \ldots, r_{\mathrm{a}, m}\right)=(v, \ldots, v)$ according to Def. 1, i.e.

$$
\begin{aligned}
\operatorname{det}\left(\mathcal{L}_{\boldsymbol{G}_{\mathrm{a}}} \mathcal{L}_{\boldsymbol{f}_{\mathrm{a}}}^{v-1} h_{\mathrm{c}, \mathrm{a}, i}(\boldsymbol{\chi})\right) \neq 0 \quad & \forall 1 \leq i \leq m \text { and } \\
\mathcal{L}_{\boldsymbol{G}_{\mathrm{a}}} \mathcal{L}_{\boldsymbol{f}_{\mathrm{a}}}^{k} \boldsymbol{h}_{\mathrm{c}, \mathrm{a}}(\boldsymbol{\chi})=\mathbf{0} & \text { for } 0 \leq k<v-1 .
\end{aligned}
$$

Note that, as the augmented dynamics may be designed freely, it is also possible to choose the dynamics (15) with more than $m v$ states and outputs with relative degrees greater then $v$. If, however, the total relative degree is smaller than the number of states, internal dynamics occur, which require an additional examination of stability. By choosing (15) according to Ass. 5, the total relative degree is $r_{\mathrm{a}}=m v$, which avoids additional internal dynamics. I/O-linearization of (15) yields the input transformation

$$
\begin{aligned}
\boldsymbol{u}_{\mathrm{a}}^{(v)} & =\mathcal{L}_{\boldsymbol{G}_{\mathrm{a}}} \mathcal{L}_{\boldsymbol{f}_{\mathrm{a}}}^{v-1} \boldsymbol{h}_{\mathrm{c}, \mathrm{a}}(\boldsymbol{\chi}) \boldsymbol{u}_{\mathrm{c}}+\mathcal{L}_{\boldsymbol{f}_{\mathrm{a}}}^{v} \boldsymbol{h}_{\mathrm{c}, \mathrm{a}}(\boldsymbol{\chi}) \\
& =\boldsymbol{A}_{\mathrm{a}}(\boldsymbol{\chi}) \boldsymbol{u}_{\mathrm{c}}+\boldsymbol{b}_{\mathrm{a}}(\boldsymbol{\chi}) .
\end{aligned}
$$

Note that $\boldsymbol{A}_{\mathrm{a}}(\boldsymbol{\chi}) \in \mathbb{R}^{v \times v}$ is non-singular due to Ass. 5 . Combining (1) with (15) yields the augmented system

$$
\begin{gathered}
\dot{\tilde{\boldsymbol{x}}}=\tilde{\boldsymbol{f}}(\tilde{\boldsymbol{x}})+\tilde{\boldsymbol{G}}(\tilde{\boldsymbol{x}}) \boldsymbol{u}_{\mathrm{c}} \\
\tilde{\boldsymbol{f}}(\tilde{\boldsymbol{x}})=\left[\begin{array}{c}
\boldsymbol{f}(\boldsymbol{x})+\boldsymbol{G}(\boldsymbol{x}) \boldsymbol{u}_{\mathrm{a}} \\
\boldsymbol{f}_{\mathrm{a}}(\chi)
\end{array}\right], \tilde{\boldsymbol{G}}(\tilde{\boldsymbol{x}})=\left[\begin{array}{c}
\mathbf{0} \\
\boldsymbol{G}_{\mathrm{a}}(\chi)
\end{array}\right]
\end{gathered}
$$

with the state $\tilde{\boldsymbol{x}}=\left[\boldsymbol{x}^{\top}, \boldsymbol{\chi}^{\top}\right]^{\top} \in \mathbb{R}^{n+m \cdot v}$ and $\boldsymbol{u}_{\mathrm{a}}=\boldsymbol{h}_{\mathrm{c}, \mathrm{a}}(\boldsymbol{\chi})$.

Example: Augmentation by $v=1$ is achieved by a single integrator

$$
\dot{\chi}=u_{\mathrm{c}}, \quad u_{\mathrm{a}}=\chi
$$

increases the relative degree by $v=1$, and results in $\boldsymbol{A}_{\mathrm{a}}=1$ and $\boldsymbol{b}_{\mathrm{a}}=0$. It achieves a continuous control input $\boldsymbol{u}_{\mathrm{a}} \in \mathcal{C}^{0}$ and therefore a state $\boldsymbol{x} \in \mathcal{C}^{1}$.

\section{B. Corrective Control Design}

Using the augmentation (17)-(18) and the constraints (4), invariance control determines a constraint enforcing input $\boldsymbol{u}_{\mathrm{c}}$ for the augmented system. Again, this requires I/Olinearization with respect to each constraint function

$$
\begin{aligned}
h_{\mathrm{c}, i}^{\left(r_{\mathrm{a}, i}\right)}(\tilde{\boldsymbol{x}}) & =\tilde{\boldsymbol{a}}_{i}^{\top}(\tilde{\boldsymbol{x}}) \boldsymbol{u}_{\mathrm{c}}+\tilde{b}_{i}(\tilde{\boldsymbol{x}}) \\
\text { with } \tilde{\boldsymbol{a}}_{i}^{\top}(\tilde{\boldsymbol{x}}) & =\mathcal{L}_{\tilde{\boldsymbol{G}}} \mathcal{L}_{\tilde{\boldsymbol{f}}}^{r_{\mathrm{a}, i}-1} h_{\mathrm{c}, i}(\tilde{\boldsymbol{x}}), \tilde{b}_{i}(\tilde{\boldsymbol{x}})=\mathcal{L}_{\tilde{\boldsymbol{f}}}^{r_{\mathrm{a}, i}} h_{\mathrm{c}, i}(\tilde{\boldsymbol{x}}) .
\end{aligned}
$$

The new relative degree $r_{\mathrm{a}, i}$ of the output is determined by the original relative degree $r_{i}$ and by the degree of augmentation $v$.

Lemma 1: Let Ass. 3 and 5 hold. Then the I/Olinearization of (17)-(18) with respect to a single constraint function $y_{\mathrm{c}, i}=h_{\mathrm{c}, i}(\boldsymbol{x})=h_{\mathrm{c}, i}(\tilde{\boldsymbol{x}})$ from (2) and the control input $\boldsymbol{u}_{\mathrm{c}}$ has a well-defined relative degree $r_{\mathrm{a}, i}=r_{i}+v$.

Proof: With Ass. 3, the I/O-linearization of $h_{\mathrm{c}, i}(\tilde{\boldsymbol{x}})$ with respect to $\boldsymbol{u}_{\mathrm{a}}=\boldsymbol{h}_{\mathrm{c}, \mathrm{a}}(\boldsymbol{\chi})$ is given by (5). Further derivation of $h_{\mathrm{c}, i}(\tilde{\boldsymbol{x}})$ yields

$$
\begin{aligned}
h_{\mathrm{c}, i}^{\left(r_{i}+k_{i}\right)}(\tilde{\boldsymbol{x}}) & =\frac{\mathrm{d}^{k_{i}}}{\mathrm{~d} t^{k_{i}}}\left(\boldsymbol{a}_{i}^{\top}(\boldsymbol{x}) \boldsymbol{h}_{\mathrm{c}, \mathrm{a}}(\boldsymbol{\chi})+b_{i}(\boldsymbol{x})\right) \\
& =\boldsymbol{a}_{i}^{\top}(\boldsymbol{x}) \boldsymbol{h}_{\mathrm{c}, \mathrm{a}}^{\left(k_{i}\right)}(\boldsymbol{\chi})+R_{i, k_{i}}(\boldsymbol{x}, \boldsymbol{\chi})
\end{aligned}
$$

which consists of one term depending on the $k_{i}$-th derivative of $\boldsymbol{h}_{\mathrm{c}, \mathrm{a}}(\boldsymbol{\chi})$, i.e. $\boldsymbol{u}_{\mathrm{a}}$, and a résidu $R_{i, k_{i}}(\boldsymbol{x}, \boldsymbol{\chi})$ depending on the lower order derivatives $\boldsymbol{u}_{\mathrm{a}}^{(j)}, j \in\left\{0,1, \ldots, k_{i}-1\right\}$. As a result from Ass. 5, the I/O-linearization of $\boldsymbol{u}_{\mathrm{a}}$ with respect to $\boldsymbol{u}_{\mathrm{c}}$ is given by (16). This means that $\boldsymbol{u}_{\mathrm{a}}^{(v)}$ and therefore also $h_{\mathrm{c}, i}^{\left(r_{i}+v\right)}(\tilde{\boldsymbol{x}})$ are the first time derivatives, which are directly influenced by $\boldsymbol{u}_{\mathrm{c}}$, which yields

$$
h_{\mathrm{c}, i}^{\left(r_{i}+v\right)}(\tilde{\boldsymbol{x}})=\boldsymbol{a}_{i}^{\boldsymbol{\top}}(\boldsymbol{x}) \mathcal{L}_{\boldsymbol{G}_{\mathrm{a}}} \mathcal{L}_{\boldsymbol{f}_{\mathrm{a}}}^{v-1} \boldsymbol{h}_{\mathrm{c}, \mathrm{a}}(\boldsymbol{\chi}) \boldsymbol{u}_{\mathrm{c}}+R_{i, v}(\boldsymbol{x}, \boldsymbol{\chi}),
$$

where $R_{i, v}(\boldsymbol{x}, \boldsymbol{\chi})=\tilde{b}_{i}(\tilde{\boldsymbol{x}})$. Ass. 5, implies that the matrix $\boldsymbol{A}_{\mathrm{a}}(\boldsymbol{\chi})=\mathcal{L}_{\boldsymbol{G}_{\mathrm{a}}} \mathcal{L}_{\boldsymbol{f}_{\mathrm{a}}}^{v-1} \boldsymbol{h}_{\mathrm{c}, \mathrm{a}}(\boldsymbol{\chi})$ is invertible and Ass. 3 ensures that $\boldsymbol{a}_{i}^{\top}(\boldsymbol{x})$ has at least one non-zero element. Therefore $\tilde{\boldsymbol{a}}_{i}^{\top}(\tilde{\boldsymbol{x}})=\boldsymbol{a}_{i}^{\top}(\boldsymbol{x}) \boldsymbol{A}_{\mathrm{a}}(\boldsymbol{\chi})$ also has at least one non-zero element thus implying the well-defined augmented relative degree $r_{\mathrm{a}, i}=r_{i}+v$.

The corresponding invariance functions are then defined by (8) using $r_{\mathrm{a}, i}=r_{i}+v$. They determine the invariant set $\mathcal{G}(\tilde{\boldsymbol{x}})$ and the set of active constraints $\mathcal{K}(\tilde{\boldsymbol{x}})$ according to (9) and (10), respectively. Solving a constrained minimization problem yields constraint enforcing control

$$
\begin{aligned}
& \quad \boldsymbol{u}_{\mathrm{c}}=\underset{\boldsymbol{u}}{\operatorname{argmin}}\left\|\boldsymbol{u}-\boldsymbol{u}_{\mathrm{no}, \mathrm{a}}\right\|_{2}^{2} \\
& \text { s.t. } \tilde{\boldsymbol{a}}_{i}^{\top}(\tilde{\boldsymbol{x}}) \boldsymbol{u}+\tilde{b}_{i}(\tilde{\boldsymbol{x}}) \leq z_{\mathrm{c}, \mathrm{a}, i} \quad \forall i \in \mathcal{K}(\tilde{\boldsymbol{x}})
\end{aligned}
$$

where $z_{\mathrm{c}, \mathrm{a}, i}$ is determined according to (11) with the nominal pseudo input $z_{\mathrm{no}, \mathrm{a}, i}=\tilde{\boldsymbol{a}}_{i}^{\top}(\tilde{\boldsymbol{x}}) \boldsymbol{u}_{\mathrm{no}, \mathrm{a}}+\tilde{b}_{i}(\tilde{\boldsymbol{x}})$ instead of $z_{\mathrm{no}, i}$. Here, $\boldsymbol{u}_{\mathrm{no}, \mathrm{a}}$ is an augmented version of nominal control.

\section{Nominal Control Design}

It is not possible to use $\boldsymbol{u}_{\mathrm{no,a}}=\boldsymbol{u}_{\text {no }}$ as this does not achieve the desired nominal behavior due to the added dynamics. Obviously, the augmentation prevents an instantaneous switch to nominal control. Instead, a feedback control law is designed such that $\boldsymbol{u}_{\mathrm{a}} \rightarrow \boldsymbol{u}_{\mathrm{no}}$ for $t \rightarrow \infty$. We propose the following control design

$$
\boldsymbol{u}_{\mathrm{no}, \mathrm{a}}=\boldsymbol{A}_{\mathrm{a}}^{-1}(\boldsymbol{\chi})\left(\boldsymbol{u}_{\mathrm{no}}^{(v)}-\boldsymbol{b}_{\mathrm{a}}(\boldsymbol{\chi})-\sum_{j=0}^{v-1} k_{j} \boldsymbol{I}_{m} \boldsymbol{e}_{\mathrm{u}}^{(j)}\right)
$$

with $\boldsymbol{e}_{\mathrm{u}}^{(j)}=\boldsymbol{u}_{\mathrm{a}}^{(j)}-\boldsymbol{u}_{\mathrm{no}}^{(j)}, \boldsymbol{u}_{\mathrm{a}}^{(j)}=\mathcal{L}_{\boldsymbol{f}_{\mathrm{a}}}^{j} \boldsymbol{h}_{\mathrm{c}, \mathrm{a}}(\boldsymbol{\chi})$, the design parameters $k_{j} \in \mathbb{R}$, the identity matrix $\boldsymbol{I}_{m} \in \mathbb{R}^{m \times m}$ and the inverse $\boldsymbol{A}_{\mathrm{a}}^{-1}(\chi)$, which exists due to Ass. 5. The control law (22) requires the derivatives of $\boldsymbol{u}_{\text {no }}$ up to the $v$-th order.

Assumption 6: The nominal control value is $v$ times continuously differentiable, i.e. $\boldsymbol{u}_{\text {no }} \in \mathcal{C}^{v}$.

As $\boldsymbol{u}_{\text {no }}$ is a function of the desired trajectory $\boldsymbol{w}(t)$ and $\boldsymbol{x}$, which is at least $v$ times continuously differentiable due to the augmentation, Ass. 6 is equal to the condition $\boldsymbol{w}(t) \in$ $\mathcal{C}^{v}$. If $\boldsymbol{w}(t) \notin \mathcal{C}^{v}$, i.e. $\boldsymbol{u}_{\text {no }} \notin \mathcal{C}^{v}$, a different asymptotically stabilizing tracking control law has to replace (22). This has however no effect on the augmentation approach.

Lemma 2: Let (15) be controlled by $\boldsymbol{u}_{\mathrm{c}}=\boldsymbol{u}_{\mathrm{no}, \mathrm{a}}$ from (22). Let Ass. 5 and 6 hold. Then, if

$$
d(s)=\sum_{j=0}^{v-1} k_{j} s^{j}
$$

is Hurwitz, the control error $\boldsymbol{e}_{\mathrm{u}}=\boldsymbol{u}_{\mathrm{a}}-\boldsymbol{u}_{\mathrm{no}}$ is uniformly exponentially stable with respect to the origin.

Proof: Substitution of (22) in (16) yields

$$
\boldsymbol{u}_{\mathrm{a}}^{(v)}=\boldsymbol{u}_{\mathrm{no}}^{(v)}-\sum_{j=0}^{v-1} k_{j} \boldsymbol{I}_{m \times m}\left(\boldsymbol{u}_{\mathrm{a}}^{(j)}-\boldsymbol{u}_{\mathrm{no}}^{(j)}\right)
$$


and the decoupled error dynamics

$$
\boldsymbol{e}_{\mathrm{u}, i}^{(v)}=-\sum_{j=0}^{v-1} k_{j} e_{\mathbf{u}, i}^{(j)}
$$

for $i \in\{1,2, \ldots, m\}$, which represent linear time-invariant dynamics with the transfer function

$$
G_{i}(s)=\frac{1}{\sum_{j=0}^{v-1} k_{j} s^{j}} .
$$

By the Routh-Hurwitz stability criterion, the error dynamics are uniformly exponentially stable if $d(s)=\sum_{j=0}^{v-1} k_{j} s^{j}$ is a Hurwitz polynomial.

As $k_{j}, j \in\{1,2, \ldots, v-1\}$ are design parameters, they may be chosen arbitrarily to satisfy the Routh-Hurwitz criterion. Using these results, the characteristics of the augmented system (17)-(18) under nominal control are shown.

Theorem 1: Let Ass. 1, 5 and 6 hold. Then, if $\boldsymbol{u}_{\text {no }}$ stabilizes the tracking error $\boldsymbol{e}_{\mathrm{w}}=\boldsymbol{w}(t)-\boldsymbol{x}(t)$ of (1) uniformly exponentially in the origin, the tracking error of the augmented system (17)-(18) controlled by (22) is uniformly asymptotically stable.

Proof: As a result from Lemma 2, $\boldsymbol{u}_{\mathrm{a}}=\boldsymbol{u}_{\mathrm{no}}+\boldsymbol{e}_{\mathrm{u}}$ is fulfilled and $\lim _{t \rightarrow \infty} \boldsymbol{e}_{\mathrm{u}}=\mathbf{0}$. Insertion into (1) yields

$$
\dot{\boldsymbol{x}}=\boldsymbol{f}(\boldsymbol{x})+\boldsymbol{G}(\boldsymbol{x})\left(\boldsymbol{u}_{\mathrm{no}}+\boldsymbol{e}_{\mathrm{u}}\right),
$$

which is a representation of the nominally controlled dynamics with the additional input $\boldsymbol{e}_{\mathrm{u}}$. With [12, Lemma 4.6] and under Ass. 1, the nominally controlled system (1) is ISS with respect to the additional input, if its tracking error is uniformly exponentially stable for $e_{\mathrm{u}}=0$. As $e_{\mathrm{u}}$ is the output of the error dynamics (23), which are uniformly exponentially stable, the tracking error of the cascade connection of the error dynamics and (1) under nominal control is uniformly asymptotically stable according to [12, Lemma 4.7] if the nominally controlled system is ISS, i.e. if it has an exponentially stable tracking error.

Theorem 1 establishes the asymptotic stability of the nominally controlled system. As $\boldsymbol{u}_{\text {no }}$ is reached asymptotically, other performance specifications are fulfilled asymptotically.

\section{Invariance and Stability}

The goal of invariance control is to guarantee constraint enforcement. Corrective control has to render the state $\tilde{\boldsymbol{x}}$ controlled positive invariant with respect to the invariant set without destabilizing the controlled dynamics. Corrective control for the augmented system is determined using (11) and (21) and ensures that the input of the I/O-linearized integrator chain fulfills $z_{i} \leq \gamma_{i}$. By applying this input the integrator chain is rendered invariant with respect to $\Phi_{i}\left(\tilde{\boldsymbol{x}}, \gamma_{i}\right) \leq 0$ [7] and since all integrator chains are rendered invariant simultaneously, the augmented system is positive invariant with respect to the invariant set $\mathcal{G}(\tilde{\boldsymbol{x}})$. With the invariance properties established, the stability is investigated, starting with the internal dynamics.

Theorem 2: Let Ass. 1-6 hold. Let the set $\mathcal{K}_{\mathcal{I}}$ contain exactly $m$ constraints fulfilling (14). Then, the I/O-linearization of (17)-(18) with respect to these constraints yields inputto-state stable (ISS) internal dynamics.
Proof: With Ass. 3 and 5, I/O-linearization of (17)(18) with respect to $\mathcal{K}_{\mathcal{I}}$ yields a well-defined vector relative degree $\left(r_{1}+v, \ldots, r_{m}+v\right)$ and a total relative degree $r=$ $\sum_{i=1}^{m}\left(r_{i}+v\right)=\sum_{i=1}^{m} r_{i}+m v$. For $r=n+m v$, the total relative degree is equal to the number of states. In this case, the internal dynamics are ISS as there are none.

For $r<n+m v$, the internal dynamics have $n+m v-r=$ $n-\sum_{i=1}^{m} r_{i}$ states. The I/O-linearization is represented by

$$
\left[\begin{array}{l|ll}
\boldsymbol{\xi}^{\top} & \boldsymbol{\eta}^{\top}
\end{array}\right]=\left[\begin{array}{lll|l}
\ldots & \boldsymbol{\xi}_{i}^{\top} & \ldots & \tilde{\boldsymbol{\Psi}}(\tilde{x})^{\top}
\end{array}\right]
$$

where $\boldsymbol{\xi}_{i}^{\top}=\left[h_{\mathrm{c}, i}(\boldsymbol{x}) \ldots h_{\mathrm{c}, i}^{\left(r_{i}+v\right)}(\boldsymbol{x})\right] \in \mathbb{R}^{r_{i}+v}, 1 \leq i \leq m$ are the states of the integrator chains and $\eta$ the internal states. This transformation has to be a diffeomorphism, i.e. there exists an inverse transformation expressing the states $\boldsymbol{x}$ and $\chi$ as functions of $\boldsymbol{\xi}_{i}$ and $\boldsymbol{\eta}$.

The derivatives of the constraint functions yield $\sum_{i=1}^{m} r_{i}$ equations which depend only on $\boldsymbol{x}$. The remaining derivatives yield $m v$ equations depending on $\boldsymbol{x}$ and $\boldsymbol{\chi}$, i.e. from these equations $\chi$ may be determined as a function of $\boldsymbol{x}$ and $\boldsymbol{\xi}$. Therefore, the internal states $\boldsymbol{\eta}=\tilde{\boldsymbol{\Psi}}(\tilde{\boldsymbol{x}})$ have to be determined such that they complement the $\sum_{i=1}^{m} r_{i}$ equations from the constraint derivatives. According to [12, Theorem 13.1], the internal dynamics are determined by

$$
\frac{\partial \tilde{\boldsymbol{\Psi}}(\tilde{\boldsymbol{x}})}{\partial \tilde{\boldsymbol{x}}} \tilde{\boldsymbol{G}}(\tilde{\boldsymbol{x}})=0 .
$$

With $\tilde{\boldsymbol{G}}(\tilde{\boldsymbol{x}})$ from (18), the equation transforms into

$$
\frac{\partial \tilde{\boldsymbol{\Psi}}(\tilde{\boldsymbol{x}})}{\partial \boldsymbol{\chi}} \boldsymbol{G}_{\mathrm{a}}(\boldsymbol{\chi})=0
$$

For $\tilde{\boldsymbol{\Psi}}(\tilde{\boldsymbol{x}})=\boldsymbol{\Psi}(\boldsymbol{x})$, this is fulfilled as $\frac{\partial \boldsymbol{\Psi}(\boldsymbol{x})}{\partial \boldsymbol{\chi}}=0$ holds. This means that the augmented dynamics inherit the internal dynamics from I/O-linearization of (1). Therefore the derivatives and $\boldsymbol{\Psi}(\boldsymbol{x})$ define a diffeomorphism and the inverse transformation determines $\boldsymbol{x}$. As a result, choosing $\tilde{\boldsymbol{\Psi}}(\tilde{\boldsymbol{x}})=$ $\boldsymbol{\Psi}(\boldsymbol{x})$ completes the diffeomorphism (24) and the original and the augmented system have the same internal dynamics, which are ISS due to Ass. 4.

Theorem 2 shows internal stability for $\left|\mathcal{K}_{\mathcal{I}}\right|=m$, but in general $\left|\mathcal{K}_{\mathcal{I}}\right|<m$ holds.

Corollary 1: Let Ass. 1-6 hold. Then, the I/Olinearization of (17)-(18) with respect to the constraints in the set $\mathcal{K}_{\mathcal{I}}$ fulfilling (14) yields ISS internal dynamics.

Proof: For $\left|\mathcal{K}_{\mathcal{I}}\right|=m$, Theorem 2 applies and the internal dynamics are ISS. For $\left|\mathcal{K}_{\mathcal{I}}\right|=k<m, m-k$ artificial constraints $h_{\text {art }, i}=\boldsymbol{c}_{i}^{\top} \boldsymbol{x}$ are added, which fulfill

$$
\begin{aligned}
\boldsymbol{c}_{i}^{\top} \boldsymbol{c}_{i} & =1, \forall 1 \leq i \leq m-k \\
\boldsymbol{c}_{i}^{\top} \boldsymbol{c}_{j} & =0, \forall 1 \leq i, j \leq m-k, i \neq j \\
\boldsymbol{a}_{i}^{\top} \boldsymbol{c}_{j} & =0, \forall i \in \mathcal{K}_{\mathcal{I}}, 1 \leq j \leq m-k .
\end{aligned}
$$

These constraints are orthogonal to each other and to the actual constraints. They complement $\mathcal{K}_{\mathcal{I}}$ by adding the conditions $\boldsymbol{c}_{i}^{\top} \boldsymbol{u}=\boldsymbol{c}_{i}^{\top} \boldsymbol{u}_{\mathrm{no}, \mathrm{a}}$, which do not change the solution of (13) and (21) as they ensure that $\boldsymbol{u}_{\mathrm{no}, \mathrm{a}}$ is applied in unconstrained directions. With these added constraints, Theorem 2 is applicable, which means that the internal dynamics are ISS for any number of active constraints. 
Based on these results, the stability of the controlled augmented system is investigated.

Theorem 3: Let Ass. 1-6 hold. Let (1) be state controllable. Let nominal control $\boldsymbol{u}_{\text {no }}$ stabilize the tracking error $\boldsymbol{e}_{\mathrm{w}}=\boldsymbol{w}(t)-\boldsymbol{x}(t)$ of (1) uniformly exponentially. Then, the state $\tilde{\boldsymbol{x}}=\left[\boldsymbol{x}^{\top}, \chi^{\top}\right]^{\top}$ of (17)-(18) controlled by the solution of (21)

(i) fulfills $\boldsymbol{x} \in \mathcal{C}^{v}$ and

(ii) asymptotically tracks a constraint admissible trajectory.

Proof: For the proof of (i), $\tilde{\boldsymbol{h}}(\boldsymbol{x})=\boldsymbol{x}$ is introduced. As $\dot{\tilde{\boldsymbol{h}}}(\boldsymbol{x})=\dot{\boldsymbol{x}}=\boldsymbol{f}(\boldsymbol{x})+\boldsymbol{G}(\boldsymbol{x}) \boldsymbol{u}_{\mathrm{a}}$ holds, the input $\boldsymbol{u}_{\mathrm{a}}$ appears in the first derivative of each $\tilde{h}_{i}(\boldsymbol{x})$ at the earliest. As (1) is assumed to be state controllable, the input influences each $x_{i}$, but not necessarily in the first derivative. Therefore, each $\tilde{h}_{i}(\boldsymbol{x})$ has a relative degree of $\tilde{r}_{i} \geq 1$ with respect to $\boldsymbol{u}_{\mathrm{a}}$. With Lemma $1, \tilde{h}_{i}(\boldsymbol{x})$ has a relative degree $\tilde{r}_{\mathrm{a}, i}=$ $\tilde{r}_{i}+v \geq 1+v$ with respect to the input of the augmented system $\overline{\boldsymbol{u}}_{\mathrm{c}}$. As the function $\tilde{h}_{i}(\boldsymbol{x})=x_{i}$ is at least $1+v$ times differentiable for all states $1 \leq i \leq n$, it is at least $v$ times continuously differentiable, i.e. $\boldsymbol{x} \in \mathcal{C}^{v}$ holds.

For the proof of (ii), the cases of no active constraints and an arbitrary number of active constraints are considered separately. If no constraint is active or the constraints are fulfilled by nominal control, the augmented system is under nominal control and the tracking is asymptotically stable by Theorem 1 . Otherwise $\mathcal{K}_{\mathcal{I}}$ contains $k \leq m$ constraints, which require corrective action $\gamma_{i}$, i.e. $\Phi_{i}\left(\bar{x}, \gamma_{i}\right) \geq 0$ holds. For $\Phi_{i}\left(\boldsymbol{x}, \gamma_{i}\right)>0, \gamma_{i}<0$ is applied, which reduces the state values in the integrator chain and therefore eventually results in $\Phi_{i}\left(\boldsymbol{x}, \gamma_{i}\right)<0$ and the application of $z_{\text {no,a }, i}$. For $z_{\text {no,a }, i}>0$, the integrator states and $\Phi_{i}\left(\boldsymbol{x}, \gamma_{i}\right)$ increase again, resulting in infinitely fast switching between the nominal and the corrective input. As both are directed towards $\Phi_{i}\left(\boldsymbol{x}, \gamma_{i}\right)=0$, the dynamics are asymptotically stabilized in $\Phi_{i}\left(\boldsymbol{x}, \gamma_{i}\right)=0$, i.e. in a constraint admissible state trajectory. For $z_{\text {no,a, } i}<0$ the state retreats further from the constraint and nominal control is applied for an extended amount of time, thus stabilizing the integrator chain by Theorem 1 . In addition there are $m-k$ integrator chains corresponding to actual and artificial constraints, which are controlled by nominal control and which are therefore asymptotically stable by Theorem 1 . With Theorem 2 and Corollary 1, the internal dynamics of the I/O-linearized system are ISS. As the controlled I/Olinearized system consists of asymptotically stable integrator chains and ISS internal dynamics, the entire augmented system (17)-(18) controlled by the solution of (21) has an asymptotically stable tracking error [12, Lemma 13.2] and the tracked trajectory is constraint admissible as the states remain within the invariant set.

Note that, although ideal sliding at the constraints requires infinitely fast switching, the control law may also be implemented with a finite switching frequency and occurring chattering effects may be handled according to [13].

\section{NUMERICAL EXAMPLE}

In this section, the derived approach is illustrated using the model of an inverted pendulum with a velocity constraint.

A. Setup

We consider the nonlinear system

$$
\begin{aligned}
\dot{\boldsymbol{x}} & =\boldsymbol{f}(\boldsymbol{x})+\boldsymbol{g}(\boldsymbol{x}) u_{\mathrm{a}} \\
\boldsymbol{f}(\boldsymbol{x}) & =\left[\begin{array}{c}
x_{2} \\
\frac{g}{l} \sin \left(x_{1}\right)-\frac{m \cos \left(x_{1}\right) \sin \left(x_{1}\right)\left(l x_{2}^{2}+g \cos \left(x_{1}\right)\right)}{l\left(m \sin ^{2}\left(x_{1}\right)+M\right)}
\end{array}\right] \\
\boldsymbol{g}(\boldsymbol{x}) & =\left[\begin{array}{ll}
0 & \frac{\cos \left(x_{1}\right)}{l\left(m \sin ^{2}\left(x_{1}\right)+M\right)}
\end{array}\right]^{\top},
\end{aligned}
$$

with the angle to the upright position $x_{1}$, the angular velocity $x_{2}$, the length $l$, the gravity constant $g$ and the masses of the pendulum and the cart $m$ and $M$. The nominal control goal of keeping the pendulum upright is achieved by the feedback control law $u_{\text {no }}=-k_{P} x_{1}-k_{D} x_{2}$. The constraint $h_{\mathrm{c}}(\boldsymbol{x})=x_{2}-\dot{\theta}_{\max }$ defines a constant velocity bound $\dot{\theta}_{\max }$. In order to achieve a continuous control input, the augmentation (19) is used. As the above control law is continuously differentiable, augmented nominal control is given by

$$
u_{\mathrm{no}, \mathrm{a}}=\dot{u}_{\mathrm{no}}-k_{0}\left(u_{\mathrm{a}}-u_{\mathrm{no}}\right)
$$

according to (22). Derivation of $h_{\mathrm{c}}(\boldsymbol{x})$ yields a well-defined relative degree $r_{a}=2$ for $x_{1} \neq \pm \frac{\pi}{2}+k, k \in \mathbb{Z}$ and

$$
\tilde{a}=\frac{\cos \left(x_{1}\right)}{l\left(m \sin ^{2}\left(x_{1}\right)+M\right)} .
$$

The calculation of the expression for $\tilde{b}$ from (16) is straightforward but omitted here because of space limitations. The invariance function $\Phi(\boldsymbol{x}, \gamma)$ is defined according to (8)

$$
\Phi(\tilde{\boldsymbol{x}}, \gamma)= \begin{cases}h_{\mathrm{c}} & \text { for } \dot{h}_{\mathrm{c}} \leq 0 \\ -\frac{\dot{h}_{\mathrm{c}}^{2}}{2 \gamma}+h_{\mathrm{c}} & \text { else }\end{cases}
$$

and corrective control is then determined with $z_{\mathrm{c}}$ from (11).

$$
u_{\mathrm{c}}= \begin{cases}u_{\mathrm{no}, \mathrm{a}} & \text { for } \Phi(\tilde{\boldsymbol{x}}, \gamma)<0 \\ \frac{z_{\mathrm{c}}-\tilde{b}}{\tilde{a}} & \text { for } \Phi(\tilde{\boldsymbol{x}}, \gamma) \geq 0\end{cases}
$$

The model is implemented in Matlab/Simulink using the parameters in Table I. An Euler solver determines the solution with a step size of $T_{A}=1 \cdot 10^{-5} \mathrm{~s}$. Two constraints with $\dot{\theta}_{\max , 1}$ and $\dot{\theta}_{\max , 2}$ are implemented and $\dot{\theta}_{\max , 1}$ is switched off at $t=1 \mathrm{~s}$. The augmented approach is compared to standard invariance control [7] for constraints with $r=1$.

\section{B. Results}

The results generated by standard and augmented invariance control using $k_{0}=1$ and $k_{0}=5$ in the augmented nominal control law (22) are depicted in Fig. 4. The invariance function in Fig. 4a has a non-positive value and the angular velocity in Fig. 4b lies below the constraints for all times, thus showing that both the standard and the augmented invariance control enforce the constraints. In addition, both approaches are able to preserve the stability of the nominal 
TABLE I: Model parameters

\begin{tabular}{lll}
\hline System parameters & $\boldsymbol{x}(0)$ & {$\left[-5 \frac{\pi}{180} \mathrm{rad}, 0 \mathrm{rad} / \mathrm{s}\right]^{\top}$} \\
& $l ; g$ & $0.2 \mathrm{~m} ; 9.81 \mathrm{~m} / \mathrm{s}^{2}$ \\
& $m ; M$ & $0.1 \mathrm{~kg} ; 1 \mathrm{~kg}$ \\
Constraint & $\dot{\theta}_{\max , 1}$ & $1 \frac{\pi}{180} \mathrm{rad} / \mathrm{s}$ \\
& $\dot{\theta}_{\max , 2}$ & $1.5 \frac{\pi}{180} \mathrm{rad} / \mathrm{s}$ \\
Nominal control & $k_{P} ; k_{D}$ & $10 \mathrm{~N} ; 1 \mathrm{~N} \mathrm{~s}$ \\
Standard invariance & $\gamma$ & $-0.11 / \mathrm{s}$ \\
Augmented invariance & $\gamma$ & $-101 / \mathrm{s}^{2}$ \\
\hline
\end{tabular}

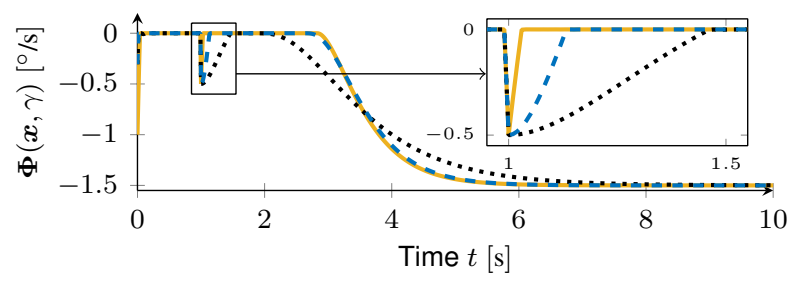

(a) Invariance function

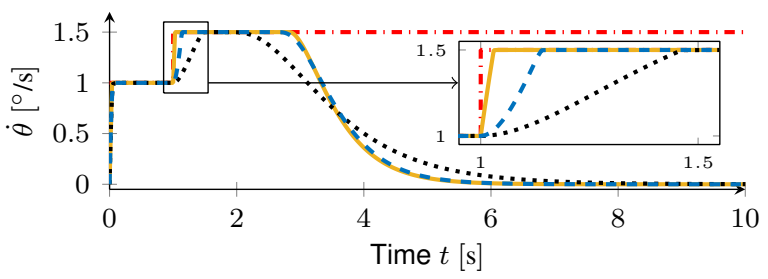

(b) Angular velocity with -..- active constraints

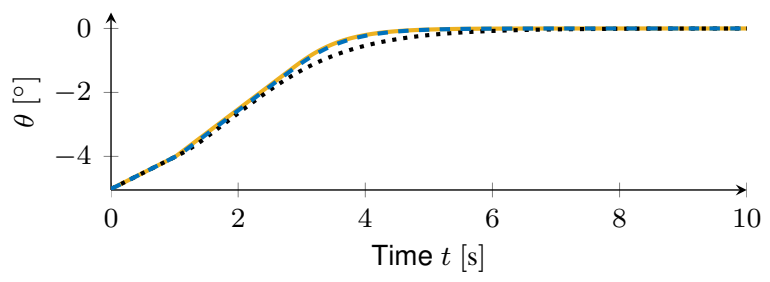

(c) Angular position

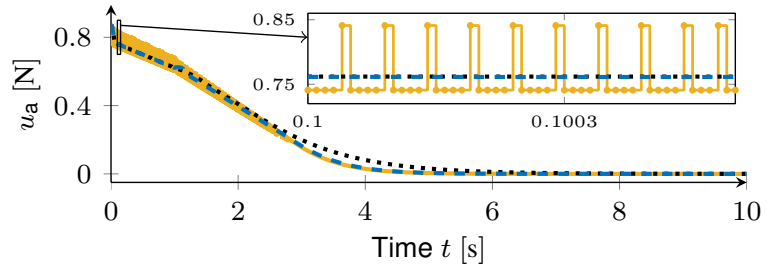

(d) Control input

Fig. 4: Results for the pendulum with $—$ standard invariance control and augmented invariance control for $\cdots \cdots k_{0}=$ 1 and $-=k_{0}=5$

control law, since the angular position in Fig. 4c approaches the desired value zero despite the constraints. This also indicates the stability of the internal dynamics. The difference between the approaches is visible in the control input in Fig. 4 d. Whenever a constraint is active, i.e. $\Phi(\boldsymbol{x}, \gamma) \geq 0$, the control input of the standard invariance control is fast and discontinuously switching between nominal and corrective control, whereas the augmented invariance control provides a continuous control input. Furthermore, whenever nominal control is applied, i.e. $\Phi(\boldsymbol{x}, \gamma)<0$, standard invariance control immediately applies the nominal control input. It reaches the constraints and the goal angle fastest as depicted in Fig. $4 \mathrm{a}-4 \mathrm{c}$. For increasing values of $k_{0}$ the tracking of the augmented invariance control improves and approaches the performance of standard invariance control.

\section{CONClusion}

In this work, a novel control approach is introduced, which combines methods from invariance control with an augmented version of a control affine dynamical system in order to achieve constraint enforcement as well as desired dynamics of the control input and smoothness of the system states. The approach is shown to guarantee the adherence to constraints while reaching the nominal control task asymptotically in unconstrained directions. An illustrating numerical example emphasizes the results.

\section{ACKNOWLEDGMENT}

This work was supported by the EU Horizon2020 project RAMCIP, under grant agreement no. 643433 and by the EU Seventh Framework Programme FP7/2007-2013 within the ERC Starting Grant Control based on Human Models (conhumo) under grant agreement no. 337654.

\section{REFERENCES}

[1] D. S. Nunes, P. Zhang, and J. S. Silva, "A Survey on Human-in-theLoop Applications Towards an Internet of All," IEEE Communications Surveys Tutorials, vol. 17, no. 2, pp. 944-965, 2015.

[2] E. Rimon and D. Koditschek, "Exact Robot Navigation Using Artificial Potential Functions," IEEE Transactions on Robotics and Automation, vol. 8, no. 5, pp. 501-518, 1992.

[3] D. Mayne, J. Rawlings, C. Rao, and P. Scokaert, "Constrained model predictive control: Stability and optimality," Automatica, vol. 36, no. 6, pp. 789-814, 2000.

[4] D. Angeli and E. Mosca, "Command Governors for Constrained Nonlinear Systems," IEEE Transactions on Automatic Control, vol. 44, no. 4, pp. 816-820, 1999.

[5] S. Prajna and A. Rantzer, "Convex Programs for Temporal Verification of Nonlinear Dynamical Systems," SIAM Journal on Control and Optimization, vol. 46, no. 3, pp. 999-1021, 2007.

[6] A. Ames, J. Grizzle, and P. Tabuada, "Control Barrier Function based Quadratic Programs with Application to Adaptive Cruise Control," in Proc. of the IEEE 53rd Annual Conference on Decision and Control (CDC), Dec 2014, pp. 6271-6278.

[7] J. Wolff and M. Buss, "Invariance Control Design for Nonlinear Control Affine Systems under Hard State Constraints," in Proc. of the NOLCOS'2004 Symposium on Nonlinear Control Systems, Stuttgart, Germany, 2004, pp. 711-716.

[8] M. Scheint, J. Wolff, and M. Buss, "Invariance Control in Robotic Applications: Trajectory Supervision and Haptic Rendering," in Proc. of the American Control Conference (ACC), Seattle, USA, 2008, pp. 1436-1442.

[9] M. Kimmel and S. Hirche, "Invariance Control with Time-varying Constraints," 2016, submitted to ECC 2016.

[10] A. Isidori, Nonlinear Control Systems, 3rd ed. Springer-Verlag, Berlin, 1995.

[11] S. P. Boyd, Convex Optimization, L. Vandenberghe, Ed. Cambridge University Press, 2004.

[12] H. Khalil, Nonlinear Systems, 3rd ed. Prentice Hall, 1996.

[13] M. Kimmel and S. Hirche, "Invariance Control with Chattering Reduction," in Proc. of the IEEE 53rd Annual Conference on Decision and Control (CDC), 2014. 